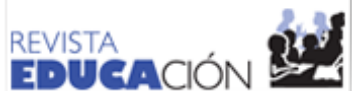

Revista Educación

ISSN: 0379-7082

ISSN: 2215-2644

revedu@gmail.com

Universidad de Costa Rica

Costa Rica

\section{Perspectiva estudiantil del modelo pedagógico flipped classroom o aula invertida en el aprendizaje del inglés como lengua extranjera}

Aguayo Vergara, Margarita; Bravo Molina, Mario; Nocetti de la Barra, Alejandra; Concha Sarabia, Lucy; Aburto Godoy, Raquel

Perspectiva estudiantil del modelo pedagógico flipped classroom o aula invertida en el aprendizaje del inglés como lengua extranjera

Revista Educación, vol. 43, núm. 1, 2019

Universidad de Costa Rica, Costa Rica

Disponible en: http://www.redalyc.org/articulo.oa?id=44057415007

DOI: https://doi.org/10.15517/revedu.v43i1.31529

Esta obra está bajo una Licencia Creative Commons Atribución-NoComercial-SinDerivar 3.0 Internacional. 
Perspectiva estudiantil del modelo pedagógico flipped classroom o aula invertida en el aprendizaje del inglés como lengua extranjera

Students' perspective on flipped classroom pedagogical approach in the learning of english as a foreign language

Margarita Aguayo Vergara

Universidad del Bio-Bio, Chile

DOI: https://doi.org/10.15517/revedu.v43i1.31529

rmaguayo@ubiobio.cl

Mario Bravo Molina

Universidad del Bio-Bio, Chile

mbravo@ubiobio.cl

Alejandra Nocetti de la Barra

Universidad Católica de la Santísima Concepción (UCSC),

Chile

anocetti@ucsc.cl

Lucy Concha Sarabia

Universidad del Bio-Bio, Chile

lucyconcha@gmail.com

Raquel Aburto Godoy

Universidad del Bío-Bio, Chile

raburto@ubiobio.cl

\section{Resumen:}

La adquisición de una segunda lengua constituye un aspecto fundamental en la sociedad globalizada del siglo XXI. La política pública nacional en Chile optó por promover el aprendizaje del idioma inglés. En ese contexto, el objetivo del estudio fue describir la percepción de los estudiantes acerca de la incorporación de la metodología flipped classroom en la asignatura de Inglés Comunicacional en las carreras de Arquitectura e Ingeniería Civil Química. Se realizó un estudio descriptivo de tipo diseño transversal con muestreo no probabilístico intencional. Se diseñó y validó un cuestionario mediante juicio de expertos en educación, el cual contempló tres dimensiones: el aprendizaje antes de la clase, aprendizaje en el aula y la valoración de la metodología. Los datos fueron analizados mediante estadística descriptiva y los resultados muestran que el estudiantado valora disponer de los videos con los contenidos, los cuales podían revisar previo a la clase presencial. Asimismo, ellos se percibieron como protagonistas de su aprendizaje y el docente como un guía y facilitador. En conclusión, la metodología diversifica los momentos para aprender, mejora el conocimiento previo y optimiza un proceso de formación centrado en el estudiante.

PALABras ClaVE: lengua extranjera, aprendizaje activo, aula invertida, educación superior.

\section{Abstract:}

The acquisition of a second language constitutes a fundamental aspect of globalized society in the 21 st century. The learning of the English language is promoted through the national public policy of education. In this context, the objective of the study was to describe the students' perception about the implementation of the flipped classroom methodology in a communicative English course. The study was carried out with undergraduate students from Architecture and Civil Chemical Engineering careers. The study was descriptive with a cross-sectional design, and an intentional non-probabilistic sample. A questionnaire was designed and validated through expert judgment. It considered three dimensions: learning before class, learning in the classroom, and assessing the methodology. The data were analyzed using descriptive statistics. The results show that students value that they can watch the content videos and they can review them prior to the face-to-face class. Also, the students perceived that the teacher/student roles changed in the class. The teacher became a guide and facilitator and students became more active in their own learning process. 
In conclusion, the flipped classroom methodology diversifies the learning spaces, improves prior knowledge and optimizes the formation process to allow a student-centered approach.

KEYWORDS: Foreign Language, Active Learning, Flipped Classroom, Higher Education.

\section{INTRODUCCIÓN}

En la sociedad del conocimiento, el aprendizaje de un idioma distinto a la lengua madre constituye un aspecto fundamental puesto que expande las fronteras de estudio y trabajo. Según datos entregados por el Cambridge English Language Assessment hay cerca de 400 millones de nativos de habla anglosajona y según estadísticas mundiales de la Organización de la Naciones Unidas para la Educación, la Ciencia y la Cultura (UNESCO, 2010), se señala al idioma inglés como la segunda lengua que más se practica. Esto pone de manifiesto la importancia de incluir en el plan de formación de los profesionales la adquisición de este idioma, constituyéndose en un desafío para la mayoría de las instituciones de educación superior a nivel nacional.

La meta formativa anterior, exige investigación en el campo de los procesos de enseñanza, pues los resultados de aprendizaje en esta área todavía resultan deficientes en los niveles en que tradicionalmente se enseña el idioma inglés. En general, aunque se han desarrollado innovaciones didácticas y paulatinamente se han diversificado los recursos de apoyo al aprendizaje del inglés, todavía el estudiantado chileno no demuestra el nivel de competencia comunicativa establecidas por el Ministerio de Educación. Además, esto resulta relevante en el marco de la implementación del modelo educativo de la Universidad del Bío-Bío (2008), el cual privilegia la formación profesional basada en un currículum por competencias que se compromete con la apropiación de una segunda lengua. Este modelo hace un cambio de paradigma y coloca a los estudiantes en el centro del proceso, con modelos que estimulan su curiosidad, creatividad, habilidades, trabajo en equipo y conocimientos adquiridos fuera del aula.

En el contexto anterior, el objetivo del estudio fue describir la percepción de los estudiantes acerca de la incorporación de la metodología flipped classroom en la asignatura de Inglés Comunicacional en las carreras de Arquitectura e Ingeniería Civil Química, en una universidad pública en la región del Biobío en Chile.

\section{ANTECEDENTES PRÁCticos}

La enseñanza del inglés como lengua extranjera monopolizó el espacio curricular nacional en los niveles de educación básica y media, siendo obligatoria desde quinto año básico según lo establecido por el Ministerio de Educación (Decreto 40,1996). Considerando lo anterior, el Ministerio establece en las bases curriculares del año 2012 que el propósito de esta asignatura es que los alumnos aprendan el idioma y lo utilicen como una herramienta para desenvolverse en situaciones comunicativas simples de variada índole y, principalmente, para acceder a nuevos conocimientos y aprendizajes y responder a las demandas de comunicación global a través de los medios y tecnologías actuales.

Cabe reconocer que a nivel nacional se han desarrollado esfuerzos para mejorar la competencia comunicativa en inglés, como por ejemplo el Programa Inglés Abre Puertas (PIAP) del Ministerio de Educación, que fue creado en el año 2004. La misión de este programa es "mejorar el nivel de inglés que aprenden los(as) estudiantes de $5^{\circ}$ año básico a $4^{\circ}$ año medio, a través de la definición de estándares nacionales para el aprendizaje del inglés, una estrategia de desarrollo profesional docente y del apoyo a los(as) profesores(as) de inglés en las salas de clases" (Decreto 81). No obstante lo anterior, International Education First (2014), demuestra que en Chile existe un bajo nivel del manejo del idioma inglés, observándose una brecha entre géneros, dando una ventaja en el uso del idioma a favor de las mujeres. Los resultados del SIMCE inglés 2010, muestran esta deficiencia y así el 82\% de los estudiantes secundarios no logran el nivel B1 establecido en las bases curriculares. ${ }^{[1]}$ 
La iniciativa anterior resulta coherente con el desarrollo de una política pública que promueve el aprendizaje de idiomas extranjeros como mecanismo de desarrollo económico, cultural y social de un país. A nivel de la enseñanza superior, también se ha fomentado la incorporación de asignaturas de inglés en los planes de estudio de los diferentes programas de formación profesional, tanto en pregrado como en posgrado. Tales iniciativas reconocen este saber cómo una competencia clave para el desempeño de los futuros profesionales del siglo XXI. Dada la importancia de ello, resulta relevante desarrollar estudios en torno al impacto que tiene la inclusión de nuevas metodologías, los recursos y la gestión del aula sobre la adquisición de este idioma en el contexto universitario.

De la implementación de esta metodología a nivel de educación superior, destacan investigaciones como de la Universidad de Antioquia en Medellín, Colombia (Gómez, 2016) donde se llevó a cabo un estudio del uso del flipped classroom en la pronunciación del inglés con futuros docentes de lenguas. Los resultados sugieren que, si bien no hubo diferencias estadísticamente significativas en los desempeños de la pronunciación entre los estudiantes del grupo control respecto a los del experimental, sí pudo evidenciarse una valorización al uso de videos y plataformas tecnológicas, la optimización eficiente del tiempo y la centralidad en el estudiante durante el proceso de enseñanza-aprendizaje.

Otro estudio que aplicó esta metodología en la educación superior fue en la Facultad de Formación del Profesorado y Educación de la Universidad de Oviedo, España, en un programa de refuerzo para la enseñanza del inglés (Lemmer, 2016), con el objetivo de mejorar la motivación de los estudiantes y reducir su ansiedad y las dificultades derivadas del aprendizaje de idiomas. También se aplicó esta metodología en la Universidad Central del Ecuador para el desarrollo de la competencia léxica en inglés, demostrándose la relevancia del modelo y de la propuesta metodológica de intervención logrados a través del uso apropiado de colocaciones en contextos reales como respuesta a la aplicación de estrategias efectivas para el trabajo cooperativo y para la resolución de problemas (Altamirano y Mercedes, 2016).

Hung (2015) utilizó el método del aula invertida con estrategias de WebQuest para el aprendizaje de inglés comunicacional en una universidad de Taiwán. El objetivo consistió en estudiar el rendimiento académico, actitudes frente al aprendizaje y los niveles de participación. Se encontró que este modelo era más eficaz que el de una clase tradicional porque los estudiantes demuestran mayor grado de compromiso con las tareas fuera del aula ayudándolos a alcanzar mejores resultados de aprendizaje. Dado el impacto positivo en cómo los estudiantes percibieron el ambiente dentro del aula y la participación en el proceso de aprendizaje, se reconoce la importancia de continuar investigando acerca de la influencia de esta metodología en distintas áreas disciplinares.

\section{RefERENTES CONCEPtUALES}

Las nuevas tecnologías siguen transformando la enseñanza universitaria en diferentes disciplinas, proponiendo modos los cuales difieren de la forma tradicional. El uso pertinente de las TIC y los procesos de innovación que las incorporan cobran mayor importancia al considerar que el estudiantado de hoy se caracteriza por pertenecer a la denominada generación de "nativos digitales" (Prensky, 2010), quienes se distinguen por poseer habilidades tecnológicas. Esto justifica el desarrollo de iniciativas orientadas a la innovación pedagógica con la incorporación de las TIC (Moreno, López y Cruz, 2014). En dicho contexto, cabe destacar que las actividades didácticas acompañadas de tecnologías resultan motivadoras en el aprendizaje de un idioma extranjero, pues promueven la interacción entre los estudiantes, ofrecen la posibilidad de tener contacto con el uso real y funcional del idioma y constituyen una gran fuente de material auténtico que mejora la competencia comunicativa (Cziko, 2009; Warschauer y Whittaker, 2002).

De acuerdo con Salinas (2004), la incorporación de las TIC en los procesos de enseñanza superior requieren de transformaciones de la práctica que se relacionan con el rol del docente, rol del alumno, metodologías e implicancias institucionales. Si se analizan los primeros años de utilización de las TIC 
en la formación profesional, se observa su centralidad en la innovación técnica para crear entornos de aprendizaje basados en la tecnología. Recientemente, el foco transitó hacia el alumno, implicando una sólida fundamentación metodológica.

Desde una perspectiva institucional, los objetivos de integrar TIC tenderían a ofrecer un espacio formativo que recoja las necesidades individuales y sociales, distinguiéndose por su carácter flexible al combinar trabajo y estudio, reconocer diferentes ritmos y estilos de aprendizaje, para lo cual los educadores debieran diseñar escenarios pedagógicos que fomenten el desarrollo de las competencias requeridas en la era digital.

En este escenario, destaca el modelo pedagógico denominado flipped classroom o clase invertida, término acuñado por Bergman y Sams (2012) para referirse al proceso de enseñanza que invierte la forma en que se trabajan los contenidos de una asignatura. Estos dos profesores de química motivados por apoyar el aprendizaje de los estudiantes que por diversas razones no asistían a clase, impulsaron el uso de la filmación de las clases y se dieron cuenta que este recurso no solo favoreció a los inasistentes, sino también a quienes asistían regularmente, porque les permitía revisar, profundizar los contenidos y resolver sus dudas.

Para estos autores, el flipped classroom constituye un enfoque integral ya que articula la instrucción directa -representada por el video- y las estrategias constructivistas dentro del aula que mejoran la reconstrucción conceptual, el compromiso e implicación del estudiante con el contenido del curso, a partir de la interacción entre el alumnado y una contextualización del aprendizaje.

Los principales dirigentes de la Red de Aprendizaje Flipped (2014), anunciaron una definición concreta del flipped learning y los "cuatro pilares" que lo sustentan y que derivan de la sigla en inglés "FLIP":

Flexible environment - Ambientes flexibles: Los educadores crean espacios adaptables donde los alumnos eligen cuándo y dónde aprenden. Además, los educadores que invierten sus clases, son flexibles en sus expectativas en los tiempos de aprendizaje y en la evaluación de los estudiantes.

Learning culture - Cultura de aprendizaje: El modelo de aprendizaje flipped cambia deliberadamente la instrucción hacia un enfoque centrado en el alumno, en el que el tiempo de clase se dedica a explorar los temas con mayor profundidad y crear más oportunidades de aprendizaje. Los estudiantes participan activamente en la construcción del conocimiento, pues participan y evalúan su aprendizaje de una manera que puede ser personalmente significativa.

Intentional content - Contenido intencional: Los educadores piensan continuamente sobre cómo pueden utilizar este modelo para ayudar a los estudiantes a desarrollar la comprensión conceptual y la fluidez de procedimiento. Los profesores emplean contenido intencional para maximizar el tiempo de clase con el fin de adoptar métodos y estrategias activas de aprendizaje centrados en el estudiante.

Professional educator - educador profesional: los educadores profesionales observan continuamente sus alumnos, proporcionándoles retroalimentación relevante en cada momento así como evaluación de su trabajo. Los educadores profesionales son reflexivos en su práctica, interactúan entre sí para mejorar la calidad de su docencia, aceptan la crítica constructiva y toleran el "caos controlado en sus aulas".

Por consiguiente, la exposición y explicación de contenidos ocurre fuera del aula por medio de herramientas tecnológicas, que pueden ser el video o el podcast, o simplemente el uso guiado de la web. Así, en el aula, las actividades prácticas como ejercitación, transferencia, construcción y aplicación de teorías y concepto, usualmente concebidas como tareas, pueden ser ejecutadas a través de métodos interactivos de trabajo colaborativo, aprendizaje basado en problemas y realización de proyectos (Coufal, 2014; Martínez, Esquive y Castillo, 2014).

Además, este modelo pedagógico de acuerdo con Tourón y Santiago (2015) constituirá una oportunidad de desarrollo profesional porque insta a los académicos a reflexionar sobre su enseñanza, incrementando las oportunidades para que los estudiantes internalicen y apliquen los contenidos disciplinares en lugar de desarrollar tareas orientadas a la reproducción memorística del contenido. 
Los fundamentos teóricos que justifican la clase inversa son principalmente la teoría del conflicto cognitivo de Piaget, y el aprendizaje cooperativo basado en la Zona de Desarrollo Próximo de Vygotsky (Avello y Gómez, 2010). El proceso de enseñanza y aprendizaje se concibe a través de un trabajo colaborativo e interactivo y de construcción por parte del estudiante, quien es responsable de su propio aprendizaje; mientras que el docente facilita un ambiente constructivista, con tareas significativas, en donde los conocimientos previos son el punto de partida.

Estas teorías afirman que el aprendizaje significativo solo ocurre cuando el estudiante interactúa activamente con los materiales de aprendizaje y se involucra con la recepción, integración y comunicación de información y técnicas (Moroney 2013). En palabras de Weimer (2013) el conocimiento no puede ser transferido, sino que deben ser los estudiantes quienes construyan el significado de dicho conocimiento conformándose como parte central del proceso de aprendizaje.

$\mathrm{Al}$ incrementar el tiempo de aprendizaje individual fuera de la clase, el aula invertida establece "un marco que garantice que los estudiantes reciban una educación personalizada adaptada a sus necesidades individuales" (Bergmann y Sams, 2012, p.6). El rol del profesor cambia, se acentúan los procesos de retroalimentación, dedica más tiempo a la diversidad en la clase, es decir, atiende las necesidades particulares de los estudiantes. Además, diseña actividades de aprendizaje para el aula que privilegian la aplicación de conceptos y la ejercitación de habilidades (Tourón y Santiago, 2015). En síntesis, el profesor cumple un rol de monitor y/o guía de los estudiantes en su aprendizaje, promoviendo que este surja desde la interacción en el aula, de modo que se estimule el desarrollo cognitivo. Asimismo, favorece la experimentación e indagación y la consolidación del conocimiento mediante la selección y/o diseño de videos u otro recurso audiovisual.

Actualmente, la metodología flipped classroom evidencia beneficios y comienza a demostrarse su efectividad en términos de mejorar el aprendizaje, aumentar la motivación y la autonomía académica (Sidhu, et al., 2014). Así, el aula invertida parece estar recibiendo cada vez una mayor atención en el ámbito educativo (García, 2013). Además, es evidente su expansión a diferentes contextos educativos del mundo como: Estados Unidos, Australia, Europa (Herreid y Schiller, 2013) y en algunos países de América Latina. Junto con lo anterior, se ha producido un gran avance en cuanto a tecnología y uso de dispositivos móviles que han facilitado la implementación de esta.

La oportunidad que ofrece el flipped classroom cuando se aplica a la enseñanza del inglés como lengua extranjera se contrapone a la enseñanza basada en un currículo predominantemente gramatical y centrado más en el profesor, el cual dificulta la interacción comunicativa en el aula pues cuando los estudiantes participan en una comunicación real, utilizan sus estrategias naturales para la adquisición del idioma lo que les permitirá aprender a usarlo (Gass, 2013). Así, bajo este modelo los estudiantes aprenden en casa la gramática y el vocabulario como las herramientas necesarias para comunicarse en clase; es decir, poner en práctica lo aprendido con la ayuda de sus pares y del profesor, lo que permite fortalecer las habilidades de producción del idioma inglés.

Además, el flipped classroom en la enseñanza del idioma inglés permite una mejor gestión del tiempo para que los estudiantes resuelvan dudas, realicen ejercicios en grupo y prácticas colaborativas que favorezcan el desarrollo de la competencia comunicativa. La parte presencial se convierte en un espacio para el desarrollo de la habilidad comunicativa mediante la ejercitación y la experiencia (Rodríguez et ál., 2016).

Es imposible pensar hoy en la enseñanza del inglés sin la incorporación de la tecnología, dada la gran cantidad de herramientas en la web 2.0. El uso de TIC permite enriquecer y favorecer los actuales enfoques de la adquisición del idioma inglés; en especial el enfoque comunicativo o funcional. Este enfoque contempla que el proceso de aprendizaje no es simplemente el dominio de la lengua, sino también su uso apropiado para propósitos comunicativos. Particularmente, uno de los objetivos de este enfoque es establecer la necesidad de desarrollar la habilidad del alumno de participar eficazmente en cualquier acto conversacional (Correa, 2015). Así, la calidad de oportunidades que se brinda al estudiante para hacer uso de la lengua va a depender del tipo de actividades que el docente planifique para el desarrollo integrado de las cuatro destrezas (hablar, 
escuchar, leer y escribir) intentando emular procesos reales de comunicación. Dentro de este enfoque surge el aprendizaje basado en tareas, como el role play, este permite a los estudiantes expresarse espontáneamente durante el proceso de la interacción comunicativa.

\section{Metodología}

\section{Tipo y diseño del estudio}

La investigación tuvo un carácter cuantitativo, en particular, se realiza un estudio exploratorio-descriptivo (Hernández, Fernández y Baptista, 2010) con un diseño transaccional, desarrollándose la medición de la variable en una sola oportunidad (Bisquerra, 2014; León y Montero, 2015). El objetivo del estudio fue describir la percepción de los estudiantes acerca de la incorporación de la metodología flipped classroom en la asignatura de Inglés Comunicacional en las carreras de Arquitectura e Ingeniería Civil Química, en una universidad pública de Chile.

\subsection{Sujetos}

La unidad de análisis de la investigación correspondió a estudiantes que cursan la asignatura de Inglés Comunicacional IV de carácter obligatorio, pertenecientes a las Facultades de Ingeniería y de Arquitectura, Construcción y Diseño, de la Universidad del Bío-Bío, Chile (Campus Concepción).

Se seleccionaron mediante muestreo no probabilístico 56 estudiantes y en la Tabla 1 se pueden apreciar la composición por género de los participantes del estudio.

TABLA 1

Distribución de la muestra según características personales y académicas

\begin{tabular}{clc}
\hline Género del & & \\
estudiante & Hombre & $57,1 \%$ \\
& 32 & $42,9 \%$ \\
& Mujer & \\
& 24 & 46,4 \\
Programa & & $\%$ \\
de estudio & Arquitectura & 53,6 \\
& 26 Ingeniería Civil & $\%$ \\
\hline
\end{tabular}

Elaboración propia con base en una totalidad de $\mathbf{n}=56$ casos

\subsection{Instrumento}

La variable del estudio correspondió a la percepción acerca del impacto que tiene la metodología flipped classroom sobre la naturaleza y condiciones del aprendizaje del idioma antes y durante la clase. Conforme a lo anterior, se diseñó y validó un cuestionario, por medio de Juicio de Expertos en educación, para explorar la percepción en las siguientes dimensiones: el aprendizaje antes de la clase, aprendizaje en el aula y la valoración de la metodología.

Siguiendo a Cea (1996) se inició el proceso de operacionalización de la variable y a partir de los indicadores se formularon 24 ítems con opciones de respuesta tipo Likert, Muy en desacuerdo -2: En desacuerdo- 3: De acuerdo - 4: Muy de acuerdo. 
Posteriormente, se sometió el instrumento a una evaluación de su validez mediante el procedimiento de Juicio de Expertos. Se contactó a siete académicos de universidades de la región del Biobío y Metropolitana con experiencia en la metodología flipped classroom y se les solicitó evaluar la coherencia entre cada pregunta y las dimensiones respectivas. Se consideró que la pregunta es válida para medir el indicador de la variable en estudio si obtenía un 70\% de acuerdo. Después de aplicar el instrumento a los participantes del estudio, se calculó el coeficiente de Cronbach y se obtuvo un valor de 0,94 que de acuerdo con J. Ruiz (2009) constituye una alta confiabilidad. En otras palabras, se esperaría que los resultados en una segunda aplicación debieran alcanzar valores similares. Además, se calculó la confiabilidad para cada una de las dimensiones, logrando un valor de 0,88 en la dimensión Aprendizaje fuera de la clase, 0,84 en Aprendizaje dentro del aula y 0,89 en la dimensión Valoración de la Metodología.

\subsection{Diseño de la investigación}

Cabe señalar que esta investigación se desarrolló en el contexto de un proyecto de investigación en docencia de la Universidad del Bío-Bío (código 141226 ID/doc) orientado a la innovación de metodologías activas para la enseñanza del idioma inglés. En particular, se estudió la implementación del modelo pedagógico flipped classroom en las asignaturas de Inglés Comunicacional IV, en las cuales se desarrollaron las cuatro habilidades para la competencia comunicativa: escuchar, hablar, leer y escribir. Se trabajó durante el segundo semestre del año académico, de agosto a diciembre 2016.

La implementación del modelo pedagógico contempló tres fases que se describen a continuación:

La fase inicial de planificación de la enseñanza correspondió a la selección de las unidades con contenidos gramaticales de mayor complejidad y que serían abordadas con la metodología flipped classroom. Además, en esta fase se seleccionaron videos según su pertinencia didáctica para ser visualizados por el estudiante previo a la clase.

La segunda fase fue la selección de las plataformas con que se trabajó el modelo pedagógico: Moodle y EdPuzzle. En la primera el estudiante encuentra todos los recursos de apoyo a la asignatura (programa, unidades, rúbricas, eventos, etc.). En la plataforma EdPuzzle se alojaron los videos personalizados por el docente, con preguntas abiertas y selección múltiple, asociadas a los primeros niveles de la taxonomía de Bloom, que guiaban la comprensión del contenido léxico y gramatical. Cabe subrayar que, esta plataforma permite el monitoreo y retroalimentación de los procesos de aprendizaje fuera de la clase y para esta experiencia piloto se utilizaron videos de corta duración tomados principalmente de la plataforma Youtube.

En la tercera fase se realizó la socialización del modelo pedagógico con los estudiantes: se presentó la metodología, sus objetivos, la modalidad de trabajo y la descripción de los roles y tareas.

El aula invertida se implementó en la cuarta fase. Se diseñaron las instrucciones para que los estudiantes visualizaran un video semanalmente antes de la clase presencial, y desarrollaran las actividades asociadas al contenido presentado en él. Se insertaron link de páginas con ejercicios de tipo drill, autocorregidos, los cuales daban claves al estudiante sobre lo que debía repasar en mayor profundidad. Del mismo modo, la plataforma permitió comprobar si el estudiante practicaba regularmente o no.

El aula invertida liberó tiempo de la clase para que los docentes planificaran actividades de interacción que favorecieron el enfoque comunicativo. Durante el desarrollo de la clase, se dedicaron los quince minutos iniciales a retroalimentar y aclarar dudas sobre los contenidos gramaticales presentados en el video. Posteriormente, se realizan actividades para desarrollar destrezas productivas del idioma inglés, se aplica el contenido de forma activa y colaborativa (ejercicios en parejas y/o en grupos pequeños). Al cierre de la clase se evaluó formativamente las tareas de aprendizaje en que se demuestra la apropiación y aplicación de los contenidos. 
En la fase final se desarrolló la evaluación de la experiencia del trabajo con flipped classroom mediante una obtención de datos cuantitativos y cualitativos. Así, se aplicó una encuesta a los estudiantes al finalizar el semestre y además, se desarrollaron dos focus group, uno con participación de estudiantes y otro con profesores.

El análisis de los datos se realizó con el programa estadístico SPSS versión 23 y correspondió a un análisis estadístico descriptivo de las dimensiones de la percepción de la implementación del flipped classroom. En particular, se utilizan tablas de frecuencias y se desarrollan comparaciones descriptivas mediante el uso de tablas de contingencias, centradas en la comparación de la percepción entre hombre y mujeres y el plan de estudio.

\section{Resultados, ANÁLISIS Y DisCUSIÓN}

Conforme a los objetivos del estudio, en la primera parte se exponen los resultados referidos a la percepción de aprendizaje que promueve la metodología fuera del aula. Luego, se presentan los hallazgos referidos al rol del profesor y de los estudiantes durante el aprendizaje en la clase y finalmente, se describen los resultados referidos a la valoración de la metodología.

\subsection{Aprendizaje fuera de la clase}

En cuanto a la percepción del aprendizaje fuera de la clase cuando se utiliza la metodología flipped classroom, la tabla 2 presenta los ítems que forman parte de esta dimensión del cuestionario. Además, el instrumento incluye los ítems referidos a la utilización del video como recurso didáctico central de la metodología implementada. 
TABLA 2

Enunciados Dimensión: Aprendizaje antes de la clase y recursos

\begin{tabular}{l|l}
\hline Preguntas & \\
\hline 1 & $\begin{array}{l}\text { El material audiovisual es coherente con el contenido } \\
\text { de la clase }\end{array}$ \\
\hline 2 & $\begin{array}{l}\text { La duración del material audiovisual que vemos } \\
\text { fuera de la clase es adecuado }\end{array}$ \\
\hline 3 & $\begin{array}{l}\text { El material audiovisual que revisamos fuera de la } \\
\text { clase es motivador }\end{array}$ \\
\hline 5 & $\begin{array}{l}\text { El material audiovisual que revisamos -bajo esta } \\
\text { metodología- fuera de la clase está gráficamente } \\
\text { bien diseñado }\end{array}$ \\
\hline 6 & $\begin{array}{l}\text { El material audiovisual que revisamos fuera de la } \\
\text { clase es comprensible }\end{array}$ \\
\hline 7 & $\begin{array}{l}\text { Noto que con la metodología flipped classroom me } \\
\text { preparo más para ir a clases }\end{array}$ \\
\hline 8 & $\begin{array}{l}\text { Con la metodologia flipped classroom aprendo más } \\
\text { fuera de la clase }\end{array}$ \\
\hline 9 & $\begin{array}{l}\text { Loto que con la metodología flipped classroom } \\
\text { busco más instancias para practicar lo aprendido } \\
\text { fuera de la clase }\end{array}$ \\
\hline 10 & $\begin{array}{l}\text { El material audiovisual - propio de esta metodología- } \\
\text { me permite revisar los contenidos cuantas veces } \\
\text { estime conveniente }\end{array}$ \\
\hline 11 & $\begin{array}{l}\text { Me doy cuenta que con la metodología flipped } \\
\text { classroom reviso más el material fuera de la clase }\end{array}$ \\
\hline 12 & $\begin{array}{l}\text { La metodologia flipped classroom aumentó el tiempo } \\
\text { que empleo para comprender el contenido fuera del } \\
\text { aula }\end{array}$ \\
\hline $\begin{array}{l}\text { Percibo que con esta metodología tengo más } \\
\text { seguridad para hablar inglés }\end{array}$ \\
\hline 5
\end{tabular}

Elaboración propia

En la tabla 3 y la tabla 3.1 se presentan los porcentajes de acuerdo y desacuerdo frente a ítems que conforman la dimensión Recurso y Aprendizaje antes de la clase.

TABLA 3

Resumen frecuencias dimensión Recurso y Aprendizaje antes de la clase

\begin{tabular}{lllllll}
\cline { 2 - 7 } & \multicolumn{1}{l}{ Íems } & & & & \\
\hline & P1 & P2 & P3 & P4 & P5 & P6 \\
\hline \%MD+ED & $1,8 \%$ & $10,7 \%$ & $18,2 \%$ & $10,7 \%$ & $10,8 \%$ & $26,8 \%$ \\
\hline \%MA+DA & $98,2 \%$ & $89,3 \%$ & $81,8 \%$ & $89,3 \%$ & $89,2 \%$ & $73,2 \%$ \\
\hline
\end{tabular}

TABLA 3.1

Continuación de Resumen frecuencias dimensión Recurso y Aprendizaje antes de la clase

\begin{tabular}{|c|c|c|c|c|c|c|}
\hline & \multicolumn{6}{|l|}{ Ítems } \\
\hline & P7 & P8 & P9 & P10 & P11 & P12 \\
\hline$\% \mathrm{MD}+\mathrm{ED}$ & $26,8 \%$ & $32,1 \%$ & $3,6 \%$ & $35,7 \%$ & $30,3 \%$ & $26,8 \%$ \\
\hline$\% \mathrm{MA}+\mathrm{DA}$ & $73,2 \%$ & $67,9 \%$ & $96,4 \%$ & $64,3 \%$ & $69,7 \%$ & $73,2 \%$ \\
\hline
\end{tabular}

Elaboración propia con base en una totalidad de $n=56$ casos 
De acuerdo con el modelo flipped classroom, previo a la clase los estudiantes visualizan videos para apropiarse del contenido y aplicarlo en el aula. Al respecto, se puede observar una percepción positiva por parte del estudiantado, ya que pueden detener el video, adelantarlo y atrasarlo cuantas veces lo deseen, permitiendo con ello una mejor comprensión del contenido y un ajuste de la metodología a los distintos ritmos y estilo de aprendizaje de los estudiantes, lo cual según Bergmann y Sams (2012) se logra dado que el flipped classroom promueve una enseñanza más personalizada y se atienden las necesidades particulares de los estudiantes en clase (Tourón y Santiago, 2015).

Por otro lado, un 89,3\% manifiesta que la duración de los videos utilizados es adecuada. No obstante, se evidenció una diferencia entre hombres y mujeres. Estas últimas reconocen sólo en un 20,8\% la pertinencia de la extensión de los videos.

Además el 98,2\% plantea que el recurso es coherente con los contenidos de la clase y el 89,2\% reconoce que el contenido de los videos es comprensible y por consiguiente, el recurso utilizado favorece procesos de aprendizaje. En este sentido, es importante destacar que el estudiantado manifiesta que el uso de este recurso aumenta la motivación por aprender inglés $(81,8 \%)$, pero nuevamente se observan diferencias entre hombres y mujeres, las que en un 26,1\% desestima la idea anterior. Asimismo, se encontró que los estudiantes de Arquitectura en un $28 \%$ no reconocen los videos como motivadores para aprender. El estudiantado valora positivamente el diseño gráfico del material audiovisual, alcanzando un $89,3 \%$ de acuerdo.

Una de las características que distingue la metodología flipped classroom es la diversificación de los espacios para el aprendizaje y por ello, interesó observar la percepción de los estudiantes respecto al aprendizaje antes de la clase. Cabe destacar que el mayor acuerdo se presentó frente a la valoración positiva de disponer del material audiovisual para su revisión reiterada de acuerdo a las necesidades individuales (96,4\%). Los encuestados reconocen que aumentó la preparación previo a la clase en comparación con las metodologías tradicionales (73,2\%). Esto coincide con Hsiu-Ting Hung (2015), quien propone que esta metodología significa un mayor compromiso con su preparación, lo cual refleja una clara diferencia con las asignaturas que se desarrollan bajo una metodología tradicional de la enseñanza del inglés.

Cuando se realiza un análisis por género llama la atención que solo el 50\% de las mujeres reconoce que revisa el video antes de asistir a la clase de inglés y del mismo modo, se observó que los estudiantes de Arquitectura en un porcentaje similar $(53,8 \%)$ revisa el material previo a la clase presencial. Esto resulta relevante de considerar porque se vincula con la motivación intrínseca por aprender.

Respecto a la percepción sobre el aprendizaje, los estudiantes encuestados notaron que con esta metodología hubo un incremento del aprendizaje en comparación con otras experiencias metodológicas tradicionales $(73,2 \%)$. No obstante, se reiteran diferencias en la percepción del aprendizaje entre hombres y mujeres, observándose que un $58,3 \%$ de las mujeres afirma que aumentó lo que aprenden fuera de la clase. Por otra parte, el estudiantado percibe que aumenta el tiempo para comprender y ejercitar el contenido (69,7\%).

Además, los estudiantes destacaron que esta metodología acentúa la práctica del idioma de modo independiente $(67,9 \%)$, pero en un análisis por programa de formación se encontraron diferencias en desmedro de los estudiantes de arquitectura, quienes practican menos lo aprendido (46,2\%). Los participantes evidencian que esta metodología aumenta la seguridad para hablar un idioma extranjero dado que el trabajo en casa no se hace para reforzar conceptos adquiridos en clase, sino para prepararse para la clase.

\subsection{Aprendizaje dentro del aula}

A continuación, en la tabla 4, se presentan los ítems que forman parte de la dimensión "Aprendizaje en el aula" que se refieren a aspectos tales como el rol del estudiante, docente, proceso de retroalimentación, entre otros. 
TABLA 4

Enunciados Dimensión: Aprendizaje en el Aula

\begin{tabular}{l|l}
$\begin{array}{l}\text { Preguntas } \\
13\end{array}$ & $\begin{array}{l}\text { Me doy cuenta que con la metodologia flipped } \\
\text { classroom, mi rol en el aula es más protagónico }\end{array}$ \\
\hline 14 & $\begin{array}{l}\text { Noto que con esta metodología planteo más } \\
\text { preguntas en la clase }\end{array}$ \\
\hline 15 & $\begin{array}{l}\text { Percibo que con esta metodología el rol del } \\
\text { profesor es de facilitador del aprendizaje dentro } \\
\text { de la clase }\end{array}$ \\
\hline 16 & $\begin{array}{l}\text { Noto que con la metodología flipped classroom se } \\
\text { plantean más dudas en la clase }\end{array}$ \\
\hline 17 & $\begin{array}{l}\text { Con esta metodología la retroalimentación es más } \\
\text { frecuente fuera y dentro la clase }\end{array}$ \\
\hline 18 & \begin{tabular}{l} 
Con esta metodología aprendo haciendo en clases \\
\hline
\end{tabular}
\end{tabular}

Elaboración propia

TABLA 5

Resumen frecuencias Aprendizaje en el Aula

\begin{tabular}{lllllll} 
& \multicolumn{7}{c}{ Ítems } & \multicolumn{7}{c}{} \\
\cline { 2 - 6 } & P13 & P14 & P15 & P16 & P17 & P18 \\
\hline \%MD+ED & $26,8 \%$ & $35,7 \%$ & $8,9 \%$ & $25,0 \%$ & $16,1 \%$ & $17,9 \%$ \\
\hline \% MA+DA & $73,2 \%$ & $64,3 \%$ & $91,1 \%$ & $75,0 \%$ & $84,0 \%$ & $82,1 \%$ \\
\hline
\end{tabular}

Elaboración propia con base en una totalidad de $n=56$ casos

De acuerdo con la literatura el modelo pedagógico -flipped classroom- demanda mayor responsabilidad por parte del estudiante, quien adquiere un rol distinto en su proceso de aprendizaje. Los resultados muestran en la tabla 5 que un $73,2 \%$ de los encuestados declaran tener un rol más activo dentro del aula y un $82,1 \%$ valoran aprender haciendo en clase, debido principalmente a que la metodología propicia clases experienciales o prácticas. Esto es coherente con la propuesta de Rodríguez, de Jubera, Campión y de Luis (2016), quienes sugieren que la metodología favorece la ejercitación y la experiencia durante la clase. Además los estudiantes de ingeniería perciben una mayor participación lo cual se refleja al plantear dudas, pero no ocurre lo mismo con quienes cursan la carrera de arquitectura, sólo el $55 \%$ plantea dudas en clase.

Al comparar las diferencias de género, se visualiza que las mujeres en un $62.5 \%$ tienen un rol menos activo que los hombres con un $81.2 \%$.

Uno de los aspectos relevante del flipped classroom es la retroalimentación permanente tanto por el docente como entre pares. Un $84 \%$ de los estudiantes valora las instancias que les permiten corregir sus errores, al recibir retroalimentación inmediata por el docente, quien dispone de un tiempo más adecuado para explicar los contenidos más complejos y atender a las necesidades individuales, aspectos que coinciden con los autores de esta metodología (Bergmann y Sams, 2012).

Ambos géneros destacan como beneficioso que el rol del profesor sea de un agente facilitador del aprendizaje al utilizar esta metodología (91,1\%). Este calificativo compartido por la mayoría de los participantes del estudio acentúa un cambio radical en la noción de las prácticas de enseñanza, es decir, hacer posible el aprendizaje mediante un mayor protagonismo del estudiante. Dado que el estudiante recupera el centro en el proceso de enseñanza /aprendizaje, se produce en el aula un nuevo "sentir". Sienten confianza 
y motivación por aprender, el aprendizaje se vuelve más personal y situado en experiencias que lo torna más significativo y profundo.

\subsection{Valoración de la Metodología}

En relación con la valoración de la metodología por parte del estudiantado, a continuación se presentan los ítems referidos a esta dimensión en la tabla 6.

TABLA 6

Enunciados Dimensión: Valoración de la metodología

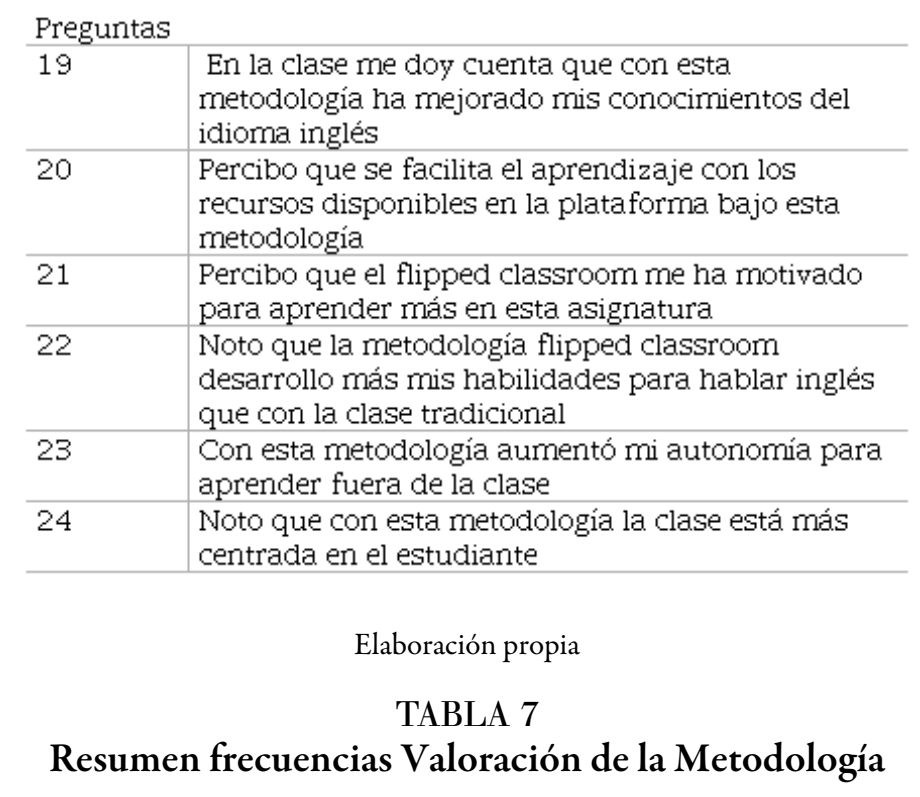

\begin{tabular}{lllllll}
\cline { 2 - 6 } & Items & \multicolumn{7}{c}{} \\
\cline { 2 - 7 } & P19 & P20 & P21 & P22 & P23 & P24 \\
\hline \%MD+ED & 17,9 & $8,9 \%$ & 25 & 28,6 & 30,4 & 7,2 \\
\hline \%MA+DA & 82,2 & 91,1 & 75 & 71,4 & 69,6 & 92,9 \\
\hline
\end{tabular}

Elaboración propia con base en una totalidad de $n=56$ casos

Incorporar el método flipped classroom en el aprendizaje del idioma inglés puede tener un papel significativo, favoreciendo aspectos como extraer del aula la explicación de los contenidos gramaticales complejos, que se pueden adquirir a través del video, para dar mayor énfasis a la práctica colaborativa de la competencia comunicativa del idioma. De lo anterior en la tabla 7, un 71,4\% de los encuestados reconocen que han mejorado sus habilidades para hablar al contrastarlo con la clase tradicional, percibiendo un progreso en su nivel inglés con el material audiovisual dispuesto en la plataforma $(91,1 \%)$.

Además, un 75\% de los encuestados declara sentirse motivado por aprender esta asignatura bajo esta metodología. Sin embargo, el nivel de motivación por carrera muestra una diferencia entre los estudiantes de ingeniería Civil Química, quienes con un $81.7 \%$ declaran sentirse más motivados con esta innovación pedagógica que los estudiantes de Arquitectura (61.5\%).

Adicionalmente, los estudiantes de Arquitectura presentan un menor desarrollo de autonomía frente a las actividades de la asignatura de inglés fuera del aula, dada las características técnicas y artísticas del programa de estudio. 
Un 92,9\% de los participantes reconoce que es una metodología más centrada en el alumno, pero solo un 69,6\% demuestra un incremento de ella en cuanto a la apropiación de contenidos fuera del aula. Al comparar la diferencia entre hombres y mujeres, sólo el 50\% de ellas reconoce un aumento de autonomía.

\section{Conclusiones}

Este estudio constituye un aporte a la investigación sobre este modelo aplicado en clases de inglés como lengua extranjera en educación superior. El objetivo fue describir la percepción de los estudiantes acerca de la incorporación de la metodología flipped classroom en la asignatura de Inglés Comunicacional en las carreras de Arquitectura e Ingeniería Civil Química, en una universidad pública de Chile.

Del análisis de los resultados, se puede concluir que los estudiantes valoraron positivamente la implementación del aula invertida, pues promueve el aprendizaje fuera del aula, mediante la observación de los videos. Además, reconocen que al adquirir herramientas a priori les da un rol más activo/ protagonista y no centrada en la trasmisión de contenidos por parte del docente. Perciben que el profesor tiene un rol de facilitador del aprendizaje, proporcionando más instancias para desarrollar la competencia comunicativa y mejorar aspectos como: gramática, pronunciación y comprensión auditiva. Así, el aprendizaje pierde su límite de tiempo y espacio y transita hacia una experiencia asincrónica. Esto hace que el ambiente del aprendizaje se vuelva más flexible, activo, colaborativo y se adapte a los tiempos y a las características de los bien llamados "nativos digitales".

De la misma forma, se puede abstraer que los estudiantes asocian este modelo pedagógico con un aprender haciendo, lo cual se condice con el constructivismo educativo el cual propone un paradigma en donde el proceso de enseñanza se percibe y se lleva a cabo como un proceso dinámico, participativo e interactivo del sujeto, de modo que el conocimiento sea una auténtica construcción del sujeto que aprende. Esto resultó importante, pues los estudiantes percibían que llegaban a la clase en igualdad de oportunidades para practicar el nuevo contenido y esto, a la vez, disminuyó la ansiedad e incrementó la confianza para practicar el idioma dentro del aula.

Junto con lo anterior, se observa que los profesores pueden adoptar paulatinamente nuevas tecnologías para proveer enseñanza asincrónica a los estudiantes, optimizando el trabajo en el aula con actividades de aplicación activa de los conceptos y el trabajo colaborativo. La implementación del aula invertida o flipped classroom implicó un cambio en la forma de planificar la enseñanza, los momentos para aprender, los roles en el proceso de enseñanza /aprendizaje.

En conclusión, este estudio respalda los hallazgos anteriores e incentiva la aplicación del modelo como una posibilidad de superar algunas de las limitaciones de los modelos de enseñanza tradicionales, como las limitaciones de tiempo o la falta de motivación de los estudiantes. Este trabajo también contribuye a la creciente cantidad de publicaciones sobre la implementación del aula invertida en el aprendizaje de inglés como lengua extranjera en la educación superior.

\section{LIMITACIONES DEL ESTUDIO}

Dentro de las posibles limitaciones del presente estudio, es importante mencionar que los resultados no pueden ser generalizados dado el alcance exploratorio de este proyecto. Segundo, se recomienda el desarrollo futuro de estudios que puedan determinar con mayor contundencia los efectos que tiene dicho modelo en el rendimiento académico y el desarrollo de habilidades del inglés y no sólo la descripción de la percepción de los estudiantes respecto a la metodología flipped classroom. Finalmente, un aspecto que no se contempló en esta investigación fue el desarrollo de un pilotaje para dar mayor solidez al proceso de validación del instrumento, cuestión que debería ser mejorada en investigaciones posteriores. 


\section{REFERENCIAS}

Altamirano, M. y Mercedes, I. (2016). Uso de actividades basadas en el modelo flipped classroom para el desarrollo de la competencia léxica en inglés (Master's thesis, Quito: UCE). Recuperado de http://www.dspace.uce.edu.ec/ bitstream/25000/8866/1/T-UCE-0010-1022.pdf

Avello, R. y Gómez, V. (2010). La construcción del conocimiento y las herramientas de trabajo colaborativo en red. Una aproximación teórica. 5to Seminario Internacional “Docencia Universitaria”. Universidad de Cienfuegos, Cuba.

Bergmann, J. y Sams, A. (2012). Flip Your Classroom: Talk To Every Student In Every Class Every Day. Washington, DC: ISTE.

Bisquerra, R. (2014). Métodos de investigación educativa: guía práctica. Ediciones Ceac: Barcelona.

Cea, M. (1996). Metodología Cuantitativa: Estrategias y técnicas de investigación social. Madrid: Síntesis.

Coufal, K. (2014). Flipped learning instructional model: Perceptions of video delivery to support engagement in eighth grade math. Lamar University-Beaumont. Recuperado de https://search.proquest.com/docview/1612629687? pq-origsite $=$ gscholar.

Correa, M. (2015). Flipping the foreign language classroom and critical pedagogies: a (new) old trend. Higher Education for the Future, 2(2), 114-125. Recuperado de http://journals.sagepub.com/doi/ abs/10.1177/2347631115584122.

Cziko, G. (2009). Una Propuesta de Modelo de Aprendizaje de Lenguas Extranjeras y Su Aplicación a Los Estudiantes de Lenguas Extranjeras de la Universidad de Playa Ancha-Valparaíso (Chile).(2009). Recuperado de https:// gerflint.fr/Base/Chili5/cziko.pdf.

Education First. (2014). Chile y el bajo nivel de inglés. Recuperado de https://www.ef.com/cl/blog/language/chiley-el-bajo-nivel-de-ingles/.

García, A. (2013). El aula inversa: cambiando la respuesta a las necesidades de los estudiantes. Revista de la Asociación de Inspectores de Educación de España, 19, 1-8. Recuperado de https://procomun.educalab.es/gl/system/files/ posts/4084afa0-5f4f-40eb-b61b-02f7df82bad8/ase19_mono02.pdf.

Gass, S. (2013). Second language acquisition: An introductory course. Routledge.

Gómez, C. (2016). El modelo Flipped learning en la pronunciación del inglés: un estudio exploratorio con docentes en formación de lenguas extranjeras. Recuperado de http://tesis.udea.edu.co/handle/10495/5243.

Hernández, R., Fernández, C. \& Baptista, P. (2010). Metodología de la Investigación. McGraw-Hill, México.

Herreid, C. \& Schiller, N. (2013). Case studies and the flipped classroom. Journal of College Science Teaching, 42(5), 62-66.

Hung, H. (2015). Flipping the classroom for English language learners to foster active learning. Computer Assisted Language Learning, 28(1), 81-96. Recuperado de https://www.learntechlib.org/p/153565/.

The Flipped Learning Network. (2014). Los cuatro pilares del Flipped Learning. Recuperado el 07 de octubre de 2018 de https://www.theflippedclassroom.es/los-cuatro-pilares-del-flipped-learning-los-conoces/

Lemmer, N. (2016). El aprendizaje inverso como programa de refuerzo para la enseñanza obligatoria en la clase de lengua extranjera inglés. Recuperado de http://digibuo.uniovi.es/dspace/handle/10651/37716

León, O. y Montero, I. (2015). Métodos de Investigación en Psicología y Educación, $4^{\circ}$ Edición. España: McGraw-Hill.

Martínez, W., Esquivel, I. y Castillo, J. (2014). Aula invertida o modelo invertido de aprendizaje: Origen, sustento e implicaciones. Los Modelos Tecno-Educativos, revolucionando el aprendizaje del siglo XXI, 137-154.

Ministerio de Educación, (2012). Bases Curriculares. Recuperado de https:// www.curriculumenlineamineduc.cl/605/articles 21319_programa.pdf

Moreno, P., López, F. y Cruz, M. (2014). Portafolio digital: un nuevo formato de aprendizaje. Recuperado de http:// dialnet.unirioja.es/servlet/articulo?codigo $=4943180$.

Moroney, S. (2013). Flipped teaching in a college algebra classroom: An action research project. Recuperado de http:// scholarspace.manoa.hawaii.edu/handle/10125/27140 
Prensky, M. (2010). Nativos e Inmigrantes Digitales. Recuperado de http://www.marcprensky.com/writing/ Prenskynativos\%20e\%20inmigrantes\%20digitales\%20(sek).pdf

Rodríguez, D., de Jubera, M., Campión, R. y de Luis, E. (2016). Diseño de un instrumento para evaluación diagnóstica de la competencia digital docente: formación flipped classroom. DIM: Didáctica, Innovación y Multimedia, (33), $1-15$.

Ruiz, J. (2009). La Evaluación del alumnado al incorporar las TIC. Congreso internacional sobre uso y buenas prácticas con TIC. Recuperado de http://palotic.es/wordpress/wp-content/uploads/2009/12/la-evaluacion-alincorporar-las-tic_jose_maria_ruiz_palomo.pdf.

Salinas, J. (2004): Cambios metodológicos con las TIC. Estrategias didácticas y entornos virtuales de enseñanza-aprendizaje. Bordón 56 (3-4). 469-481. Recuperado de http://mc142.uib.es:8080/rid=1K1RX87X3-25S6H65-4GJ/SALINAS,\%20J.\%20Cambios\%20metodol \%C3\%B3gicos\%20con\%20las\%20TIC.pdf

Sidhu, D., Kwan, R., Pexman, P.y Siakaluk, P. (2014). Effects of relative embodiment in lexical and semantic processing of verbs. Acta psychologica, 149, 32-39.

Tourón, J. y Santiago, R. (2015). El modelo Flipped Learning y el desarrollo del talento en la escuela. Revista de Educación, no 368, p. 196-231.

UNESCO. (2010). Compendio mundial de la educación 2010: comparación de las estadísticas de educación en el mundo. Recuperado de http://unesdoc.unesco.org/images/0019/001912/191218s.pdf

Warschauer, M. y Whittaker, P. (2002). The Internet for English Teaching: Guidelines for Teachers. En J. Richards y W. Renandya (eds.) Methodology in Language Teaching: An Anthology of Current Practice. Cambridge: Cambridge University Press (Pp 368-373).

Weimer, M. (2013). Learner-centered teaching: Five key changes to practice. San Francisco: Jossey-Bass.

\section{Notas}

1 Específicamente, estas bases curriculares se orientan al logro de dos niveles. El A2 corresponde a un nivel básico superior y se usa como referencia para el nivel que debe alcanzar un estudiante al terminar $8^{\circ}$ básico. El nivel B1, equivalente a un nivel intermedio inferior, corresponde al nivel mínimo que deben alcanzar los estudiantes en IV medio

\section{BY-NC-ND}

